\title{
Differences in Factors Associated with Albuminuria according to Gender and Comorbidities of Hypertension and Diabetes
}

\author{
Miae Jang ${ }^{1}$, Sohee $\mathrm{Oh}^{2}$, Hye-Mi Noh' ${ }^{1}$, Sunyoung Chun ${ }^{1}$, Hye young $\mathrm{Oh}^{1}$, Kyung Hee Park ${ }^{1}$, Yu Jin Paek ${ }^{1}$, \\ Hong Ji Song ${ }^{1, *}$ \\ 'Department of Family Medicine, Hallym University Sacred Heart Hospital, Hallym University College of Medicine, Anyang, Korea \\ ${ }^{2}$ Department of Biostatistics, Seoul Metropolitan Government Seoul National University Boramae Medical Center, Seoul, Korea
}

\begin{abstract}
Background: This study examined the differences in factors associated with albuminuria according to gender and comorbidities of hypertension (HTN) and diabetes mellitus (DM).

Methods: We included 3,859 participants aged 20 to 79 years (55\% female) from the 5th Korea National Health and Nutrition Examination Survey. Participants were excluded if they took antihypertensive or anti-diabetic medication, had chronic renal failure, had malignant tumor, were pregnant or menstruating during the health examination, or had missing urine albumin data. Albuminuria was defined by the participant's urine albumin-creatinine ratio (uACR). Relationships between dependent and independent variables were analyzed using the Pearson's correlation test and simple linear regression. Due to possible muticollinearity, multiple linear regression analysis was used to determine whether the association between the dependent and independent variables of interest remained significant after adjustment for other potentially confounding independent variables.

Results: The variables significantly correlated with uACR were different between the genders and between subjects with HTN or DM as a comorbidity. In the multiple linear regression models, hemoglobin Alc $(\mathrm{P}=0.01)$ was positively associated with UACR in men without HTN and DM. In men with HTN or DM, systolic blood pressure and fasting glucose $(\mathrm{P}<0.01)$ were positively associated with $\mathrm{UACR}$. In women with HTN or DM, waist circumference $(\mathrm{P}=0.011)$ and gamma-glutamyl transpeptidase $(\mathrm{P}<0.001)$ were positively correlated with $\mathrm{uACR}(\mathrm{P}<0.05)$ and glucose level $(\mathrm{P}=0.019)$ was negatively correlated with $\mathrm{uACR}$.

Conclusion: The study suggested factors correlated with albuminuria were different for men and women according to comorbidities such as HTN and DM.
\end{abstract}

Keywords: Albuminuria; Gender Identity; Hypertension; Diabetes Mellitus 


\section{INTRODUCTION}

A high level of urinary albumin excretion has been known to be associated with increased risk of cardiovascular and renal diseases and eventually all-cause morbidity and mortality. ${ }^{1-4)}$ The excretion of not only clinical levels of albuminuria, but also subclinical amounts of albuminuria, was found to be associated with higher risk of cardiovascular and renal disease. ${ }^{2)}$ This risk was already apparent at levels of albuminuria currently considered to be normal in general population. ${ }^{2)}$ The urine albumin-creatinine ratio ( $\mathrm{uACR})(\mathrm{mg} / \mathrm{g})$ is defined as a ratio of urine albumin $(\mathrm{mg} / \mathrm{dL})$ and urine creatinine $(\mathrm{g} / \mathrm{dL})$. It has been used to assess urine albumin excretion because it has been shown to be a good predictor of cardiovascular morbidity and all cause mortality. ${ }^{5)}$ The excretion of subclinical amounts of albumin in the urine, known as microalbuminuria, is defined as a uACR with a cut-off point of $\geq 30$ to $<300 \mathrm{mg} / \mathrm{g}$. ${ }^{5}$ Microalbuminuria is considered to be a biomarker of endothelial dysfunction and one of the early markers of cardiovascular, cerebral, and renal diseases. ${ }^{5-7)}$ Recent studies have reported the prevalence of microalbuminuria and associated risk factors in different ethnic populations. ${ }^{8-11)}$ Previous studies of the prevalence of microalbuminuria and associated risk factors have shown some inconsistent results due to ethnic differences in participants, study design, adjusted confounding factors, and comorbidities of the study population. The threshold value of uACR was adopted from studies on the risk of development of diabetic nephropathy in diabetic patients, ${ }^{12,13)}$ and the same threshold has been used in studies among both general and nondiabetic populations. Low levels of urinary albumin excretion, below the current threshold for microalbuminuria, predicted the development of cardiovascular diseases in non-hypertensive and nondiabetic individuals. ${ }^{14)}$ Therefore, for studies on albuminuria and the associated risk factors, the differences between non-hypertensive, nondiabetic individuals and patients with clinically apparent hypertension (HTN) or diabetes should be examined. It is believed that gender is a fundamental variable in research for not only autoimmune diseases, but also non-communicable diseases. Furthermore, gender difference is not only a confounding factor, but also a disease modifier in older adults. Therefore, data from men and women should be analyzed separately. ${ }^{15-21)}$ Recently, researchers have been encouraged to study the differences between men and women not only in clinical research but also in animal and in vitro studies. ${ }^{22)}$

The objective of this study was to investigate the differences in factors associated with albuminuria according to gender and the existence of HTN and type 2 diabetes mellitus (DM) in participants who were not taking medications.

\section{METHODS}

\section{Study Population}

This study was based on data acquired in the 5th Korea National Health and Nutrition Examination Survey conducted in 2011 (KNHANES V-2). The KNHANES is a cross-sectional and nationally representative survey conducted by the Korea Centers for Disease Control and Prevention periodically since 1998 to assess the health and nutritional status in a civilian, non-institutionalized Korean population. The 5th KNHANES was conducted from 2010 to 2012; urine albumin was measured in 2011. Using a stratified, multistage probability sampling design, 3,840 households were selected and 8,055 individuals completed the health interview and health examination surveys. In total, 6,308 adults aged 20-79 years were initially included. Subjects who took antihypertensive or anti-diabetic medication, those who reported a history of chronic renal failure or any cancer, women who were pregnant or menstruating during the health examination, and those with missing urine albumin data were excluded. Finally, 3,859 participants (1,753 men and 2,106 women) were included in the study population. This study was approved by the institutional review board of Hallym University Sacred Heart Hospital (IRB approval no. 2013-I075).

\section{Measurements}

The data were collected through standardized health examinations performed in specially equipped mobile examination centers and via face-to-face interviews in households. The health survey administration sequence was as follows: intake, receipt of written informed consent, anthropometric measurement, blood sampling, and completion of the questionnaire. The standardized questionnaire collected information about age, socioeconomic characteristics, medical history and drug use, smoking habits, and other lifestyle risk factors. The anthropometric data were collected according to the standardized guidelines.

Height and weight were obtained using standardized techniques and equipment. Height was measured to the nearest 0.1 cm using a portable stadiometer (Seriter, Bismarck, ND, USA). Weight was measured to the nearest $0.1 \mathrm{~kg}$ using a Giant-150N calibrated balance-beam scale (Hana, Seoul, Korea). Body mass index (BMI) was calculated by dividing weight by the square of height $\left(\mathrm{kg} / \mathrm{m}^{2}\right)$. Waist circumference (WC) was measured to the nearest $0.1 \mathrm{~cm}$ during exhalation, using a measuring tape (SECA 200; SECA, Hamburg, Germany) at the horizontal plane midway between the inferior costal margin and the iliac crest, at the mid-axillary line. The weight to height ratio was calculated as the waist circumference in centimeters divided by the height in centimeters. The dual-energy X-ray absorptiometry scan was performed for each subject to measure the total body fat mass and total body fat percentage using fan-beam technology (Lunar Co., Madison, WI, USA). 
Systolic blood pressure (SBP) and diastolic blood pressure (DBP) was measured by standard methods using a sphygmomanometer (Baumanometer; Baum, Copiague, NY, USA) with the patient in the sitting position after a 5-minute rest period. Three measurements were obtained with a 30 -second interval. The average of the second and third measurements was used in the analysis. Pulse pressure was calculated as the difference between SBP and DBP, and when the difference was more than $40 \mathrm{~mm} \mathrm{Hg}$, it was defined as wide pulse pressure. HTN was defined as having a systolic blood pressure of $140 \mathrm{~mm} \mathrm{Hg}$ or higher, or a diastolic blood pressure of $90 \mathrm{~mm} \mathrm{Hg}$ or higher. Blood samples were collected in the morning after fasting for at least 8 hours and single-spot urine specimens were collected during the first morning void. Levels of fasting plasma glucose, hemoglobin Alc (HbAlc) and serum total cholesterol, triglyceride (TG), low density lipoprotein cholesterol, and high density lipoprotein cholesterol (HDLC) were analyzed using the Hitachi Automatic Analyzer 7600 (Hitachi, Tokyo, Japan). DM was defined as having a fasting glucose of $126 \mathrm{mg} / \mathrm{dL}$ or higher, or a HbAlc of $6.5 \%$ or higher.

Smoking was measured in pack years. Alcohol consumption was measured in g/wk. Regular exercise was defined as more than 150 minutes of moderate intensity activity or 60 minutes of vigorous intensity activity per week. Data describing medications and lifestyle were obtained from structured questionnaires.

Urine albumin and creatinine concentrations in random urine samples were measured using a turbidimetric immunoassay and colorimetric method (Hitachi Automatic Analyzer 7600). The ratio of urinary albumin to urinary creatinine was reported as the uACR in milligrams per gram of creatinine.

Estimated glomerular filtration rate (eGFR) was estimated by the Modification of Diet in Renal Disease formula (eGFR [mL/ $\left.\min / 1.73 \mathrm{~m}^{2}\right]=186 \times$ serum creatinine $[\mathrm{mg} / \mathrm{dL}]^{-1.154} \times \mathrm{age}^{-0.203} \times$ 0.742 [if female]).

\section{Statistical Analysis}

Due to the complex sampling design of the KNHANES, the weighted mean and standard error (SE), or proportion (\%) and SE of the general characteristics according to gender and HTN and DM status were calculated. The assumption of normality of the data was tested by the Shapiro-Wilk test, and a P-value greater than 0.05 indicated that the observed distribution of a

Table 1. General characteristics of study subjects

\begin{tabular}{|c|c|c|c|c|c|c|}
\hline \multirow{2}{*}{ Characteristic } & \multicolumn{3}{|c|}{ HTN $(-)$ and DM (-) $(\mathrm{N}=3,213)$} & \multicolumn{3}{|c|}{$\operatorname{HTN}(+)$ or DM $(+)^{*}(\mathrm{~N}=646)$} \\
\hline & Men $(N=1,363)$ & Women $(\mathrm{N}=1,850)$ & P-value & Men $(\mathrm{N}=390)$ & Women $(\mathrm{N}=256)$ & P-value \\
\hline Age (y) & $40.05 \pm 0.54$ & $41.78 \pm 0.42$ & 0.0021 & $44.28 \pm 0.71$ & $53.85 \pm 1.01$ & $<0.0001$ \\
\hline Waist circumference (cm) & $82.89 \pm 0.37$ & $76.19 \pm 0.29$ & $<0.0001$ & $87.78 \pm 0.69$ & $83.21 \pm 0.73$ & $<0.0001$ \\
\hline Body mass index $\left(\mathrm{kg} / \mathrm{m}^{2}\right)$ & $23.69 \pm 0.12$ & $22.73 \pm 0.11$ & $<0.0001$ & $25.23 \pm 0.22$ & $24.97 \pm 0.28$ & 0.4643 \\
\hline Body fat (\%) & $20.87 \pm 0.33$ & $32.28 \pm 0.30$ & $<0.0001$ & $22.67 \pm 0.44$ & $33.40 \pm 0.66$ & $<0.0001$ \\
\hline $\mathrm{SBP}(\mathrm{mm} \mathrm{Hg})$ & $113.92 \pm 0.35$ & $108.66 \pm 0.35$ & $<0.0001$ & $133.57 \pm 0.93$ & $140.01 \pm 1.62$ & 0.0005 \\
\hline $\mathrm{DBP}(\mathrm{mm} \mathrm{Hg})$ & $75.68 \pm 0.28$ & $71.23 \pm 0.24$ & $<0.0001$ & $91.57 \pm 0.52$ & $85.84 \pm 0.91$ & $<0.0001$ \\
\hline Pulse pressure (mm Hg) & $38.24 \pm 0.25$ & $37.43 \pm 0.23$ & 0.0138 & $42.01 \pm 0.69$ & $54.17 \pm 1.29$ & $<0.0001$ \\
\hline Serum creatinine (mg/dL) & $0.97 \pm 0.00$ & $0.71 \pm 0.00$ & $<0.0001$ & $0.97 \pm 0.01$ & $0.72 \pm 0.01$ & $<0.0001$ \\
\hline $\operatorname{eGFR}\left(\mathrm{mL} / \mathrm{min} / 1.73 \mathrm{~m}^{2}\right)$ & $94.49 \pm 0.56$ & $99.86 \pm 0.62$ & $<0.0001$ & $92.23 \pm 0.91$ & $93.02 \pm 1.32$ & 0.6174 \\
\hline uACR (mg/g Cr) & $0.88 \pm 0.07$ & $1.15 \pm 0.06$ & 0.0004 & $1.66 \pm 0.12$ & $1.86 \pm 0.18$ & 0.3425 \\
\hline Glucose (mg/dL) & $4.51 \pm 0.00$ & $4.49 \pm 0.00$ & $<0.0001$ & $4.65 \pm 0.01$ & $4.64 \pm 0.01$ & 0.6538 \\
\hline HbA1c (\%) & $5.48 \pm 0.01$ & $5.45 \pm 0.01$ & 0.0619 & $6.00 \pm 0.08$ & $6.05 \pm 0.07$ & 0.6818 \\
\hline $\mathrm{TG}(\mathrm{mg} / \mathrm{dL})$ & $4.75 \pm 0.02$ & $4.46 \pm 0.02$ & $<0.0001$ & $5.05 \pm 0.04$ & $4.81 \pm 0.04$ & 0.0001 \\
\hline $\mathrm{HDLC}(\mathrm{mg} / \mathrm{dL})$ & $3.90 \pm 0.01$ & $4.03 \pm 0.01$ & $<0.0001$ & $3.89 \pm 0.01$ & $3.98 \pm 0.02$ & $<0.0001$ \\
\hline AST (IU/L) & $3.08 \pm 0.01$ & $2.90 \pm 0.01$ & $<0.0001$ & $3.25 \pm 0.03$ & $3.10 \pm 0.03$ & $<0.0001$ \\
\hline ALT (IU/L) & $3.06 \pm 0.02$ & $2.66 \pm 0.01$ & $<0.0001$ & $3.34 \pm 0.04$ & $2.98 \pm 0.04$ & $<0.0001$ \\
\hline GGT (IU/L) & $3.41 \pm 0.02$ & $2.81 \pm 0.01$ & $<0.0001$ & $3.93 \pm 0.04$ & $3.19 \pm 0.05$ & $<0.0001$ \\
\hline Vitamin D (ng/mL) & $17.77 \pm 0.27$ & $15.96 \pm 0.23$ & $<0.0001$ & $18.46 \pm 0.37$ & $17.06 \pm 0.51$ & 0.0084 \\
\hline Medication for dyslipidemia & $49(2.88)$ & 75 (3.16) & 0.6349 & $19(3.09)$ & 25 (10.58) & 0.0014 \\
\hline Current smoking & $616(63.08 \%)$ & $123(55.89)$ & 0.0631 & $185(61.77)$ & $23(65.59)$ & 0.7210 \\
\hline Alcohol drinking (g/wk) & $115.86 \pm 5.46$ & $37.60 \pm 3.60$ & $<0.0001$ & $196.02 \pm 12.46$ & $25.32 \pm 3.86$ & $<0.0001$ \\
\hline Regular exercise & 135 (10.93) & $154(8.49)$ & 0.0607 & $29(7.14)$ & $28(10.69)$ & 0.2367 \\
\hline Menopause (women only) & - & $595(24.67)$ & - & - & $163(59.75)$ & - \\
\hline
\end{tabular}

Values are presented as mean \pm standard error or number (\%). P-values were calculated by Student t-test or chi-square test. UACR, glucose, TG, HDLC, AST, ALT, and GGT were log transformed because they were not normally distributed. The data describing medications and life style were obtained from structured questionnaires. The eGFR (mL/ $\left.\mathrm{min} / 1.73 \mathrm{~m}^{2}\right)=186 \times$ serum creatinine $(\mathrm{mg} / \mathrm{dL})^{-1.154} \times$ age $^{-0.203} \times 0.742$ (if female).

HTN, hypertension; DM, type 2 diabetes mellitus; SBP, systolic blood pressure; DBP, diastolic blood pressure; eGFR, estimated glomerular filtration rate; uACR, urine albumin-tocreatinine ratio; HbA1c, hemoglobin A1c; TG, triglyceride; HDLC, high density lipoprotein cholesterol; AST, aspartate aminotransferase; ALT, alanine transaminase; GGT, gammaglutamyl transpeptidase.

*HTN was defined as having a SBP of $140 \mathrm{~mm} \mathrm{Hg}$ or higher, or a DBP of $90 \mathrm{~mm} \mathrm{Hg}$ or higher. DM was defined as having a fasting glucose of $126 \mathrm{mg} / \mathrm{dL}$ or higher, or a HbA1C of $6.5 \%$ or higher. 
variable was not statistically different from the normal distribution. The values of TG, HDLC, aspartate aminotransferase (AST), alanine transaminase (ALT), and gamma-glutamyl transpeptidase (GGT) were log transformed because they were not normally distributed. Continuous data were expressed as means and SE, and categorical data as frequencies and SE, as appropriate. To examine the gender specific relative contribution of age and body fat percentage, subgroup analyses of men and women were performed using a linear regression model. Relationships between dependent and independent variables were analyzed using the Pearson's correlation test and simple linear regression. Next, multiple linear regression analysis was used to determine whether the association between the dependent and independent variables of interest remained significant after adjusting for other potentially confounding independent variables due to possible multicollinearity All the tests were two-sided with significance levels at a $\mathrm{P}<0.05$. All the analyses were performed using IBM SPSS Statistics ver. 20.0 (IBM Co., Armonk, NY, USA) and R ver. 2.15.2 (http://www.r-project.org).

\section{RESULTS}

There were differences in almost all metabolic parameters, body fat percentage, blood pressure, and health behaviors between men and women (Table 1). In the participants without HTN and DM, eGFR and UACR were significantly higher in women than in men; however, among those with HTN or DM, there was no difference in eGFR and UACR between women and men. Again, among those without HTN and DM, BMI and fasting glucose were significantly higher in men than in women; however, there was no difference among men and women with HTN or DM. With respect to health behaviors, the number of men who consumed alcohol was higher than that of women.

The correlation analysis showed that the variables that were significantly correlated with uACR were different between genders and those with HTN and DM (Table 2). Age was negatively correlated with uACR only in women without HTN and DM $(\mathrm{P}<0.0001)$, but there was no significant relationship between $\mathrm{uACR}$ and age in women with HTN or DM and in men. WC $(\mathrm{P}=$ $0.0039)$, SBP $(\mathrm{P}=0.0003), \mathrm{DBP}(\mathrm{P}=0.0375)$, and pulse pressure $(\mathrm{P}=0.0071)$ were positively associated with $\mathrm{UACR}$ in women

Table 2. Correlation analysis for UACR according to gender and diseases (HTN or DM)

\begin{tabular}{|c|c|c|c|c|c|c|c|c|}
\hline \multirow{3}{*}{ Variable } & \multicolumn{4}{|c|}{ HTN (-) and DM (-) } & \multicolumn{4}{|c|}{ HTN $(+)$ or DM $(+)^{*}$} \\
\hline & \multicolumn{2}{|c|}{ Men } & \multicolumn{2}{|c|}{ Women } & \multicolumn{2}{|c|}{ Men } & \multicolumn{2}{|c|}{ Women } \\
\hline & r & P-value & $r$ & P-value & $r$ & P-value & r & P-value \\
\hline Age & -0.030 & 0.3437 & -0.123 & $<0.0001$ & 0.037 & 0.4772 & -0.066 & 0.3244 \\
\hline Waist circumference (cm) & 0.050 & 0.0889 & 0.006 & 0.8442 & 0.110 & 0.0708 & 0.227 & 0.0039 \\
\hline Body mass index $\left(\mathrm{kg} / \mathrm{m}^{2}\right)$ & 0.079 & 0.0064 & 0.024 & 0.3867 & 0.071 & 0.2422 & 0.154 & 0.0323 \\
\hline Body fat (\%) & 0.066 & 0.2211 & -0.000 & 0.9763 & 0.122 & 0.2048 & 0.097 & 0.3318 \\
\hline $\mathrm{SBP}(\mathrm{mm} \mathrm{Hg})$ & 0.054 & 0.1125 & 0.022 & 0.5039 & 0.065 & 0.2761 & 0.243 & 0.0003 \\
\hline $\mathrm{DBP}(\mathrm{mm} \mathrm{Hg})$ & 0.056 & 0.1590 & 0.013 & 0.6768 & 0.086 & 0.1140 & 0.163 & 0.0375 \\
\hline Pulse pressure (mm Hg) & 0.013 & 0.7028 & 0.019 & 0.5516 & 0.010 & 0.8591 & 0.178 & 0.0071 \\
\hline Serum creatinine (mg/dL) & -0.094 & 0.0068 & -0.111 & $<0.0001$ & -0.083 & 0.2683 & -0.089 & 0.1893 \\
\hline $\operatorname{eGFR}\left(\mathrm{mL} / \mathrm{min} / 1.73 \mathrm{~m}^{2}\right)$ & 0.104 & 0.0014 & 0.151 & $<0.0001$ & 0.114 & 0.0580 & 0.097 & 0.1423 \\
\hline Glucose (mg/dL) & 0.017 & 0.5449 & 0.025 & 0.4437 & 0.223 & $<0.0001$ & 0.110 & 0.1699 \\
\hline $\mathrm{HbA1c}(\%)$ & 0.074 & 0.0154 & 0.023 & 0.3948 & 0.178 & $<0.0001$ & 0.098 & 0.2469 \\
\hline $\mathrm{TG}(\mathrm{mg} / \mathrm{dL})$ & 0.052 & 0.1378 & -0.033 & 0.2412 & 0.133 & 0.0413 & -0.060 & 0.3923 \\
\hline $\mathrm{HDLC}(\mathrm{mg} / \mathrm{dL})$ & -0.039 & 0.3001 & 0.024 & 0.3780 & -0.006 & 0.9363 & -0.094 & 0.1540 \\
\hline AST (IU/L) & 0.064 & 0.0902 & -0.030 & 0.3134 & 0.178 & 0.0019 & 0.011 & 0.8793 \\
\hline ALT (IU/L) & 0.057 & 0.1159 & -0.011 & 0.7227 & 0.125 & 0.0507 & 0.052 & 0.5293 \\
\hline GGT (IU/L) & 0.082 & 0.0102 & 0.033 & 0.2422 & 0.109 & 0.0488 & 0.049 & 0.4749 \\
\hline Vitamin D (ng/mL) & 0.087 & 0.1387 & 0.135 & 0.0009 & 0.190 & 0.0367 & 0.129 & 0.1398 \\
\hline Medication for dyslipidemia (\%) & 0.052 & 0.0567 & 0.029 & 0.3089 & 0.037 & 0.4834 & 0.053 & 0.5184 \\
\hline Current smoking (\%) & 0.085 & 0.0509 & 0.018 & 0.8296 & 0.009 & 0.8847 & 0.259 & 0.1953 \\
\hline Alcohol drinking (g/wk) & 0.037 & 0.2189 & 0.016 & 0.7130 & 0.017 & 0.7575 & 0.034 & 0.6299 \\
\hline Regular exercise (\%) & -0.015 & 0.6588 & -0.030 & 0.2938 & -0.014 & 0.7991 & -0.061 & 0.3533 \\
\hline Menopause (\%) (women only) & & & -0.064 & 0.0232 & & & -0.036 & 0.6540 \\
\hline
\end{tabular}

UACR, glucose, TG, HDLC, AST, ALT, and GGT were log transformed because they were not normally distributed. The data describing medications and life style were obtained from structured questionnaires. The eGFR $\left(\mathrm{mL} / \mathrm{min} / 1.73 \mathrm{~m}^{2}\right)=186 \times$ serum creatinine $(\mathrm{mg} / \mathrm{dL})^{-1.154} \times$ age $^{-0.203} \times 0.742$ (if female).

uACR, urine albumin-to-creatinine ratio; HTN, hypertension; DM, type 2 diabetes mellitus; SBP, systolic blood pressure; DBP, diastolic blood pressure; eGFR, estimated glomerular filtration rate; HbA1c, hemoglobin A1c; TG, triglyceride; HDLC, high density lipoprotein cholesterol; AST, aspartate aminotransferase; ALT, alanine transaminase; GGT, gammaglutamyl transpeptidase.

*HTN was defined as having a SBP of $140 \mathrm{~mm} \mathrm{Hg}$ or higher, or a DBP of $90 \mathrm{~mm} \mathrm{Hg}$ or higher. DM was defined as having a fasting glucose of $126 \mathrm{mg} / \mathrm{dL}$ or higher, or a HbA1C of $6.5 \%$ or higher. 
Table 3. Multiple linear regression analysis

\begin{tabular}{|c|c|c|c|c|c|c|c|c|}
\hline \multirow{3}{*}{ Variable } & \multicolumn{4}{|c|}{ HTN (-) and DM (-) } & \multicolumn{4}{|c|}{ HTN (+) or DM (+) } \\
\hline & \multicolumn{2}{|c|}{ Men } & \multicolumn{2}{|c|}{ Women } & \multicolumn{2}{|c|}{ Men } & \multicolumn{2}{|c|}{ Women } \\
\hline & Estimate & P-value & Estimate & P-value & Estimate & P-value & Estimate & P-value \\
\hline Age & -0.002 & 0.686 & -0.011 & 0.490 & -0.010 & 0.327 & -0.003 & 0.855 \\
\hline \multicolumn{9}{|l|}{ Blood pressure } \\
\hline $\mathrm{SBP}(\mathrm{mm} \mathrm{Hg})$ & 0.010 & 0.160 & 0.008 & 0.586 & 0.028 & 0.005 & 0.023 & 0.057 \\
\hline \multicolumn{9}{|l|}{ Anthropometric parameters } \\
\hline Body mass index $\left(\mathrm{kg} / \mathrm{m}^{2}\right)$ & 0.019 & 0.392 & -0.043 & 0.126 & & & & \\
\hline Waist circumference (cm) & & & & & 0.017 & 0.204 & 0.054 & 0.011 \\
\hline \multicolumn{9}{|l|}{ Metabolic profiles } \\
\hline Glucose (mg/dL) & & & & & 2.203 & $<0.001$ & -2.212 & 0.019 \\
\hline HbA1c (\%) & 0.543 & 0.010 & 0.632 & 0.316 & & & & \\
\hline $\mathrm{TG}(\mathrm{mg} / \mathrm{dL})$ & 0.036 & 0.806 & -0.184 & 0.569 & 0.031 & 0.874 & -0.858 & 0.234 \\
\hline GGT (IU/L) & 0.173 & 0.157 & 0.126 & 0.736 & 0.584 & 0.697 & 1.160 & $<0.001$ \\
\hline eGFR (mL/min/1.73 m²) & 0.012 & 0.015 & 0.020 & 0.019 & 0.004 & 0.647 & 0.005 & 0.665 \\
\hline \multicolumn{9}{|l|}{ Life style } \\
\hline Smoking (pack/y) & 0.290 & 0.096 & 0.178 & 0.587 & -0.063 & 0.815 & 0.982 & 0.101 \\
\hline Menopause & & & -0.264 & 0.696 & & & 0.109 & 0.837 \\
\hline Model $\mathrm{R}^{2}$ & \multicolumn{2}{|c|}{0.039} & \multicolumn{2}{|c|}{0.059} & \multicolumn{2}{|c|}{0.096} & \multicolumn{2}{|c|}{0.581} \\
\hline
\end{tabular}

Urine albumin-to-creatinine ratio, glucose, TG, and GGT were log transformed because they were not normally distributed. The data describing life style were obtained from structured questionnaires. Values are standardized $\beta$-coefficients. The eGFR $\left(\mathrm{mL} / \mathrm{min} / 1.73 \mathrm{~m}^{2}\right)=186 \times$ serum creatinine $(\mathrm{mg} / \mathrm{dL})^{-1.154} \times$ age $^{-0.203} \times 0.742$ (if female).

HTN, hypertension; DM, type 2 diabetes mellitus; SBP, systolic blood pressure; HDA1c, hemoglobin A1c; TG, triglyceride; GGT, gamma-glutamyl transpeptidase; eGFR, estimated glomerular filtration rate.

*HTN was defined as having a SBP of $140 \mathrm{~mm} \mathrm{Hg}$ or higher, or a DBP of $90 \mathrm{~mm} \mathrm{Hg}$ or higher. DM was defined as having a fasting glucose of $126 \mathrm{mg} / \mathrm{dL}$ or higher, or a HbA1c of $6.5 \%$ or higher.

with HTN and DM, but not in women without HTN and DM and in men. In the univariate analysis, BMI was correlated with uACR in men without HTN and DM $(\mathrm{P}=0.0064)$ but not in men with HTN and DM and in women. Glucose, HbAlc, and TG levels were positively correlated with UACR in men with HTN and DM, but not in women. GGT was positively correlated with uACR in men, but not in women.

Multiple linear regression models were used to assess the associations of age, SBP, anthropometric parameters, metabolic profiles, lifestyle, and uACR (Table 3 ). In men without HTN and $\mathrm{DM}, \mathrm{HbAlc}(\mathrm{P}=0.01)$ was positively associated with $\mathrm{uACR}$. In men with HTN or DM, SBP $(\mathrm{P}=0.005)$ and glucose levels $(\mathrm{P}<$ $0.001)$ were positively associated with UACR. In women with HTN or DM, WC $(\mathrm{P}=0.011)$ and GGT levels $(\mathrm{P}<0.001)$ were positively associated with $\mathrm{UACR}$, and glucose levels $(\mathrm{P}=0.019)$ were negatively associated with UACR.

\section{DISCUSSION}

Factors including blood pressure, metabolic profiles, and obesity correlated with uACR were found to be different between for men and women. In men without HTN and DM, HbAlc was found to be significantly correlated with UACR. However, blood pressure, metabolic profiles, and obesity were not correlated with UACR in women without HTN and DM. Although the level of $\mathrm{HbAlc}$ was not in the range that indicates DM, HbAlc could be one of the risk factors for albuminuria in men, but not in women. Differences between the genders were noted ac- cording to the presence of HTN and DM. In men with HTN or DM, glucose level and SBP were significantly correlated with UACR. In women with HTN or DM, WC was positively correlated with uACR, but SBP was shown to be only marginally significant. Furthermore, in women with HTN or DM, glucose and uACR were negatively correlated, although men showed a positive correlation. These findings suggest that there is a paradoxical reverse correlation between glucose and UACR in women with early HTN or DM who were not taking medications, whose blood pressure and blood glucose levels were slightly elevated. This paradoxical reverse correlation between glucose and UACR was not observed in men. Therefore, it can be hypothesized that in women with early HTN or DM, a certain compensatory mechanism in the renal proximal tubule plays a role in regulating the plasma glucose levels, and such a compensatory mechanism may occur only in women in response to estrogen. Animal studies have suggested that estrogen plays a role in the preservation of renal function in chronic kidney disease and in the protection against renal aging in cases of oxidative damage. ${ }^{23,24)}$ Estrogen levels were not measured in our study, which is a limitation of our study.

Interestingly, GGT, one of the markers of oxidative stress, was shown to have a positive correlation with UACR in the group of women with HTN or DM. This suggests that abdominal obesity and oxidative stress could be contributing to the mechanism involved in the development of albuminuria in the group of women with HTN or DM.

Previous studies on albuminuria and the associated risk fac- 
tors reported inconsistent results. UACR has been used to assess urine albumin excretion, because it has been reported to be a good predictor of cardiovascular morbidity and all cause mortality. ${ }^{5}$ The cut-off value of microalbuminuria was uACR of $\geq 30$ to $<300 \mathrm{mg} / \mathrm{g}$; this value was obtained from studies about the risk of diabetic nephropathy development in diabetic patients. ${ }^{5,12,13)}$ However, previous researchers defined microalbuminuria using the same cut-off points that were used in their study among the general or nonhypertensive, nondiabetic populations. ${ }^{8,14)}$ Using one standardized uACR value ( $\geq 30 \mu \mathrm{g} / \mathrm{mg}$ ) to define microalbuminuria may not only underestimate microalbuminuria in subjects with higher muscle mass (men) and possibly in certain racial/ethnic groups such as non-Hispanic blacks but may also overestimate it in subjects with lower muscle mass (women) because the urine creatinine concentrations differ between men and women and between different racial/ethnic groups. Some previous studies have advocated use of separate uACR cut-off points for the detection of microalbuminuria in men and women. Use of sex-specific uACR values, ( $17 \mathrm{~g} / \mathrm{mg}$ in men and $25 \mathrm{~g} / \mathrm{mg}$ in women corresponding to 30 and $31 \mathrm{~g} / \mathrm{min}$ of urine albumin excretion, respectively) for comparison of the UACR in spot urine samples to albumin excretion rates measured in timed urine specimens has been suggested. ${ }^{25)}$ Connell et al. ${ }^{26)}$ collected timed overnight urine samples from 187 diabetic and 105 control subjects and found that uACR of $4.0 \mathrm{mg} / \mathrm{mmol}(22 \mathrm{~g} / \mathrm{mg})$ correlated with an albumin excretion rate of $35 \mathrm{~g} / \mathrm{min}$ in men and $23 \mathrm{~g} / \mathrm{min}$ in women. Our study suggested that the inconsistency in the results of the previous research about albuminuria and associated risk factors could be caused by differences in the study population, including differences in gender and comorbidities.

Our study has some limitations. First, it was difficult to identify the cause-and-effect relationship between albuminuria and associated risk factors due to the cross-sectional nature of the study. Second, the inability to exclude subjects with undiagnosed renal disease or other conditions that may elevate urinary albumin excretion, such as hematuria, urinary tract infection, or other febrile illness should be considered when interpreting our data. Third, the results of urine albumin creatinine ratios were analyzed based on a single measurement. While the uACR is a good single-sample marker of increased urinary albumin excretion, a single measurement of urine albumin excretion and serum creatinine for calculating UACR and eGFR could result in misleading classifications of albuminuria and due to intra-individual variability. Unfortunately, it is not feasible to take repeated measurements in nationwide health examination studies. Lastly, data on the effects of several medications, which were used, on albuminuria were limited.

Despite these limitations, one strength of our study is the use of nationally representative date from a large population-based study. Another important aspect of the study is the separate as- sessments of characteristics of men and women in the general population, of those in the group without comorbidities such as HTN and DM, and of those with either HTN or DM. Previous studies based on participants of KNHANES from 2007 to 2010 did not include HbAlc values in their studies. However, our data included $\mathrm{HbAlc}$; therefore, the prevalence of DM was not underestimated as in previous studies.

In conclusion, we found that factors correlated with albuminuria were different according to gender and comorbidities. Therefore, studies about albuminuria and risk factors should consider gender and comorbidities.

\section{CONFLICT OF INTEREST}

No potential conflict of interest relevant to this article was reported.

\section{ACKNOWLEDGMENTS}

This research was supported by Hallym University Research Fund (HURF-2015-33).

\section{REFERENCES}

1. Gerstein HC, Mann JF, Yi Q, Zinman B, Dinneen SF, Hoogwerf B, et al. Albuminuria and risk of cardiovascular events, death, and heart failure in diabetic and nondiabetic individuals. JAMA 2001;286:421-6.

2. Hillege HL, Fidler V, Diercks GF, van Gilst WH, de Zeeuw D, van Veldhuisen DJ, et al. Urinary albumin excretion predicts cardiovascular and noncardiovascular mortality in general population. Circulation 2002; 106:1777-82.

3. Jackson CE, Solomon SD, Gerstein HC, Zetterstrand S, Olofsson B, Michelson EL, et al. Albuminuria in chronic heart failure: prevalence and prognostic importance. Lancet 2009;374:543-50.

4. Mann JF, Yi QL, Gerstein HC. Albuminuria as a predictor of cardiovascular and renal outcomes in people with known atherosclerotic cardiovascular disease. Kidney Int Suppl 2004;(92):S59-62.

5. Eknoyan G, Hostetter T, Bakris GL, Hebert L, Levey AS, Parving HH, et al. Proteinuria and other markers of chronic kidney disease: a position statement of the national kidney foundation (NKF) and the national institute of diabetes and digestive and kidney diseases (NIDDK). Am J Kidney Dis 2003;42:617-22.

6. Bakris GL, Molitch M. Microalbuminuria as a risk predictor in diabetes: the continuing saga. Diabetes Care 2014;37:867-75.

7. Mehta D, Pimentel DA, Nunez MZ, Abduljalil A, Novak V. Subclinical albuminuria is linked to gray matter atrophy in type 2 diabetes mellitus. Metabolism 2014;63:1390-7.

8. Yan L, Ma J, Guo X, Tang J, Zhang J, Lu Z, et al. Urinary albumin excretion and prevalence of microalbuminuria in a general Chinese population: a cross-sectional study. BMC Nephrol 2014;15:165.

9. Pasko N, Toti F, Strakosha A, Thengjilli E, Shehu A, Dedej T, et al. Prevalence of microalbuminuria and risk factor analysis in type 2 diabetes patients in Albania: the need for accurate and early diagnosis of dia- 
betic nephropathy. Hippokratia 2013;17:337-41.

10. Masimango MI, Sumaili EK, Jadoul M, Wallemacq P, Mubagwa DK, Makulo RJ, et al. Prevalence of microalbuminuria and diagnostic value of dipstick proteinuria in outpatients from HIV clinics in Bukavu, the Democratic Republic of Congo. BMC Nephrol 2014;15:146.

11. Kim YS, Kim HS, Oh HY, Lee MK, Kim CH, Kim YS, et al. Prevalence of microalbuminuria and associated risk factors among adult Korean hypertensive patients in a primary care setting. Hypertens Res 2013;36: 807-23.

12. National Kidney Foundation. K/DOQI clinical practice guidelines for chronic kidney disease: evaluation, classification, and stratification. Am J Kidney Dis 2002;39(2 Suppl 1):S1-266.

13. Mancia G, De Backer G, Dominiczak A, Cifkova R, Fagard R, Germano G, et al. 2007 ESH-ESC Practice Guidelines for the Management of Arterial Hypertension: ESH-ESC Task Force on the Management of Arterial Hypertension. J Hypertens 2007;25:1751-62.

14. Arnlov J, Evans JC, Meigs JB, Wang TJ, Fox CS, Levy D, et al. Low-grade albuminuria and incidence of cardiovascular disease events in nonhypertensive and nondiabetic individuals: the Framingham Heart Study. Circulation 2005;112:969-75.

15. Bidulescu A, Liu J, Hickson DA, Hairston KG, Fox ER, Arnett DK, et al. Gender differences in the association of visceral and subcutaneous adiposity with adiponectin in African Americans: the Jackson Heart Study. BMC Cardiovasc Disord 2013;13:9.

16. Ahonen T, Vanhala M, Kautiainen H, Kumpusalo E, Saltevo J. Sex differences in the association of adiponectin and low-grade inflammation with changes in the body mass index from youth to middle age. Gend Med 2012;9:1-8.

17. Herrera VL, Tsikoudakis A, Ponce LR, Matsubara Y, Ruiz-Opazo N. Sex- specific QTLs and interacting loci underlie salt-sensitive hypertension and target organ complications in Dahl S/jrHS hypertensive rats. Physiol Genomics 2006;26:172-9.

18. Khuwaja AK, Kadir MM. Gender differences and clustering pattern of behavioural risk factors for chronic non-communicable diseases: community-based study from a developing country. Chronic Illn 2010;6: 163-70.

19. Song HJ, Paek YJ, Choi MK, Lee HJ. Gender differences in the relationship between risk of hypertension and fruit intake. Prev Med 2014;67: 154-9.

20. Wang SF, Shu L, Wang S, Wang XQ, Mu M, Hu CQ, et al. Gender difference in the association of hyperuricemia with hypertension in a middle-aged Chinese population. Blood Press 2014;23:339-44.

21. Vlassoff C. Gender differences in determinants and consequences of health and illness. J Health Popul Nutr 2007;25:47-61.

22. Clayton JA, Collins FS. Policy: NIH to balance sex in cell and animal studies. Nature 2014;509:282-3.

23. Mao S, Xu H, Zou L, Xu G, Wu Z, Ding Q, et al. Estrogen preserves split renal function in a chronic complete unilateral ureteral obstruction animal model. Exp Ther Med 2014;7:1555-62.

24. Pijacka W, Clifford B, Tilburgs C, Joles JA, Langley-Evans S, McMullen S. Protective role of female gender in programmed accelerated renal aging in the rat. Physiol Rep 2015;3:(1-6). pii: e12342.

25. Warram JH, Gearin G, Laffel L, Krolewski AS. Effect of duration of type I diabetes on the prevalence of stages of diabetic nephropathy defined by urinary albumin/creatinine ratio. J Am Soc Nephrol 1996;7:930-7.

26. Connell SJ, Hollis S, Tieszen KL, McMurray JR, Dornan TL. Gender and the clinical usefulness of the albumin: creatinine ratio. Diabet Med 1994; 11:32-6. 